\title{
Changes in plasma antioxidant status following consumption of diets high or low in fruit and vegetables or following dietary supplementation with an antioxidant mixture
}

\author{
Ian R. Record*, Ivor E. Dreosti and Jennifer K. McInerney \\ CSIRO Health Sciences and Nutrition, PO Box 10041, Adelaide BC, South Australia 5000, Australia
}

(Received 1 March 2000 - Revised 18 September 2000 - Accepted 7 November 2000)

\begin{abstract}
The aim of the present study was to examine the effect of consumption of a high-fruit and vegetable diet, or a spray-dried extract of selected fruits and vegetables of high antioxidant content, on indices of antioxidant status of individuals consuming a background diet with minimal antioxidant intake. Plasma antioxidant concentrations were determined in twenty-five men following a 2-week depletion period during which they consumed self-selected lowantioxidant diets (less than three servings of fruit and vegetables with no tea, coffee, red wine or fruit juice). Following this period the volunteers consumed either a self-selected diet containing five to seven servings of fruit and vegetables/d, or $30 \mathrm{~g}$ of a spray-dried supplement designed to provide the equivalent antioxidant activity of five to seven servings of fruit and vegetables for 2 weeks in a crossover trial. Following consumption of a high-antioxidant diet for 2 weeks, plasma concentrations of ascorbic acid, $\alpha$ - and $\beta$-carotene and lutein+zeaxanthin were all significantly increased $(P<0.05)$ over the depletion period. However, concentrations of lycopene, retinol and tocopherol were not affected. Consumption of the supplement also raised the concentrations of these same antioxidants in plasma. Despite the increases in the concentrations of measured antioxidant nutrients, the 6-hydroxy-2,5,7,8-tetramethylchroman2-carboxylic acid-equivalent antioxidant capacity of plasma, as estimated by inhibition of metmyoglobin activity, was not significantly affected by any of the dietary treatments.
\end{abstract}

Antioxidants: Plasma: Dietary supplement

Over recent years considerable epidemiological evidence has been gathered to suggest an association between consumption of fruits and vegetables and a reduced risk of certain chronic diseases such as cardiovascular disease (Rimm et al. 1996), stroke (Gillman et al. 1995), hypertension (Ascherio et al. 1992), cataracts (Leske et al. 1998), macular degeneration (Seddon et al. 1994), cancers (Steinmetz \& Potter, 1996) and DNA damage (Ames, 1998). In a number of cases the benefits of fruit and vegetable consumption may be a result of the consumption of dietary fibre.

However, fruits and vegetables are also a rich source of other food factors, including vitamins, minerals and nonnutritive phytochemicals which may exhibit biological activity in a number of ways. These phytochemicals may act as antioxidants, induce enzymes, act as pro- or antioestrogens, or modulate bacterial populations in the intestine (Lampe, 1999). The antioxidant activity of fruits, vegetables and beverages is often assumed to be of greatest importance in combating a number of degenerative diseases, as free radical-related damage has been implicated in causing many of these conditions (Ames, 1998). However, the recommended daily consumption of five or more servings of fruit and vegetables (Baghurst et al. 1992) to accomplish this objective is rarely met in many Western diets. In an effort to provide a viable means of increasing the intake of nutrient and non-nutrient antioxidants we used data collected earlier (Record et al. 1998) to formulate a spray-dried plant-based supplement with high antioxidant activity. The antioxidant activity was equivalent to that contained in five to seven servings of fruit and vegetables, and was derived from a diverse range of antioxidants.

The efficacy of this supplement was compared in twentyfive volunteer males with a self-chosen dietary regimen when consumption of fruit and vegetables was encouraged $v$. a control diet based on low fruit, vegetable and polyphenol intake. The variables measured were plasma antioxidant capacity, plasma antioxidant vitamins and 
Table 1. Composition of the spray-dried antioxidant supplement ${ }^{\star}$

\begin{tabular}{lccc}
\hline Ingredient & $\begin{array}{c}\text { Measured antioxidant } \\
\text { activity (mmol/g) }\end{array}$ & $\begin{array}{c}\text { Level in supplement } \\
(\mathrm{g} / \mathrm{kg})\end{array}$ & $\begin{array}{c}\text { Antioxidant contribution } \\
(\mathrm{mmol} / \mathrm{d})\end{array}$ \\
\hline Green tea polyphenol & 7200 & $10 \cdot 6$ & 2290 \\
Turmeric & 36 & $34 \cdot 1$ & 37 \\
Capsicum & 69 & $306 \cdot 6$ & 635 \\
Carrot & $6 \cdot 4$ & $400 \cdot 6$ & 77 \\
Maize & $4 \cdot 0$ & $202 \cdot 1$ & $24 \cdot 2$ \\
Silverbeet (Beta vulgaris) & 42 & $46 \cdot 0$ & 58 \\
Total & & & 3121 \\
\hline
\end{tabular}

*Volunteers were asked to consume $30 \mathrm{~g}$ supplement/d in $2 \times 15 \mathrm{~g}$ portions for 2 weeks.

carotenoids as well as albumin and uric acid, the other main non-nutrient antioxidants in plasma.

\section{Materials and methods}

\section{Preparation of the antioxidant supplement}

Antioxidant capacities of a range of fresh fruits and vegetables were determined on water-soluble portions of a number of fruits and vegetables, herbs and spices (Record et al. 1998). From these preliminary investigations it was possible to calculate a total antioxidant capacity range derived from the ingestion of five to seven normal servings of a variety of common fruit and vegetable foods. Based on this, a number of samples were evaluated for their suitability to be spray-dried following extraction. Edible portions of capsicum, carrot, maize and silverbeet (Beta vulgaris) were trimmed from fresh produce, except maize, which was from frozen maize kernels (McCain's, Doncaster, Victoria, Australia). The edible portions were milled in a commercial mill to pass through a $12.7 \mathrm{~mm}$ screen and pressed through a $0.5 \mathrm{~mm}$ screen to remove larger fragments. Maltodextrin (Starch Australasia, Sydney, NSW, Australia) was added to the maize, capsicum and carrot $(5.5,9.2$ and $5.7 \%(\mathrm{w} / \mathrm{v})$ final concentrations respectively) to aid drying. The extracts were spray-dried in a commercial spray drier with an air inlet temperature range of $195-200^{\circ} \mathrm{C}$ and an air outlet temperature range of $95-100^{\circ} \mathrm{C}$ at a feed rate of approximately $220 \mathrm{ml} / \mathrm{min}$. All spray-dried materials were prepared by Food Science Australia (Sydney, NSW, Australia). In addition, green tea polyphenols (50\% (w/w); Wing Lee, Hong Kong) and turmeric powder (Masterfoods, Wyong, NSW, Australia) were included in the final supplement. The formulation and measured antioxidant activity of each ingredient as well as that of the final supplement, as measured by the method of Miller et al. (1993), is presented in Table 1. The final

Table 2. Measured vitamin and carotenoid composition of the spraydried antioxidant supplement and the amount provided daily in $30 \mathrm{~g}$ taken as $2 \times 15 \mathrm{~g}$ portions

\begin{tabular}{lcc}
\hline & $\mu \mathrm{g} / \mathrm{g}$ supplement & $\mu \mathrm{g}$ supplied/d \\
\hline$\alpha$-Carotene & 34.5 & 1033 \\
$\beta$-Carotene & 87.6 & 2628 \\
Lutein & 18.1 & 543 \\
Vitamin C & 2.1 & 63 \\
\hline
\end{tabular}

concentration of maltodextrin was calculated to be $6.2 \%(\mathrm{w} / \mathrm{w})$.

The supplement was analysed for the contents of several vitamins and carotenoids and these were compared with the Australian recommended dietary intake, where established (National Health and Medical Research Council, 1991). As can be seen from Table 2, the contribution of the supplement to the vitamin $\mathrm{C}$ recommended dietary intake $(40 \mathrm{mg} / \mathrm{d})$ was small. In terms of retinol equivalents (recommended intake of 500-750 retinol equivalents/d) the supplement supplied approximately 524 retinol equivalents $(0.167 \times(\beta$-carotene $+0.5 \times \alpha$-carotene $)$, as described by Holland et al. (1995), which was close to meeting the recommended daily requirements. The lutein content was also substantial; however, the supplement was found to contain only negligible amounts of lycopene, retinol and vitamin $\mathrm{E}$.

\section{Study participants and protocol}

Following a newspaper advertisement and an initial screening to exclude individuals with clinical conditions or dietary habits that could compromise or be compromised by participation in the study, twenty-five males (mean age $48 \cdot 3$ (range 25-60) years; mean BMI $24 \cdot 8(20 \cdot 1-30 \cdot 3) \mathrm{kg} /$ $\mathrm{m}^{2}$; all non-smokers) were recruited. The sample size was based on power calculations from previous studies (Miller et al. 1993) and were predicted to detect a $10 \%$ change with a power of $0 \cdot 95$. Exclusion criteria included the use of dietary supplements (vitamins, carotenoids and minerals), adherence to vegetarian or macrobiotic diets, regular consumption of medications (e.g. salicylates) known to be antioxidants, or consumption of excessive amounts of alcohol (more than three standard glasses of alcohol per d). Volunteers attended an information night where background and study details were explained, and participants then received detailed instructions in smaller groups.

Subjects were instructed to consume diets low in antioxidants (i.e. no more than one piece of fruit and two servings of vegetables per $\mathrm{d}$, and to avoid tea, coffee, red wine, fruit juices, antioxidant tablets and aspirin) for a 2week washout period before the trial. Based on previous data (Record et al. 1998), the total antioxidant capacity of the diets due to fruit and vegetable consumption should have been approximately $1 \mathrm{mmol} 6$-hydroxy-2,5,7,8-tetramethylchroman-2-carboxylic acid (Trolox) equivalents/d. Suggestions were made as to various menus and dishes that 
could be prepared. This period of 2 weeks was chosen because significant decreases in plasma ascorbic acid concentrations have been detected within this time with provision of a diet low in ascorbic acid (Samman et al. 1997) After an overnight fast, blood was withdrawn (with heparin as anticoagulant). Thereafter, half the subjects were given sachets $(30 \mathrm{~g})$ of the supplement and asked to consume this as $2 \times 15 \mathrm{~g}$ portions suspended in water on a daily basis; this amounted to $3.0 \mathrm{mmol}$ Trolox equivalents/d.

The remaining volunteers were instructed to consume a diet high in antioxidants (i.e. five to seven servings of fruit and vegetables per $\mathrm{d}$, together with fruit juices), which would be expected to provide approximately $1.7-2.4$ mmol Trolox equivalents/d. As with the low-antioxidant phase, suggestions were given as to the composition of meals and menus that would yield a high proportion of fruits and vegetables.

After a further 2 weeks, all subjects returned for repeat blood sampling. At this time those subjects in the group consuming the supplement were asked to consume $15 \mathrm{~g}$ of the supplement in water and a further blood sample was taken $1 \mathrm{~h}$ later. Volunteers consuming the high- or lowantioxidant diet were given a light breakfast of water and toast spread with margarine as a low-antioxidant comparison with the supplement, and a second blood sample was taken $1 \mathrm{~h}$ later. For the final 2 weeks before blood sampling, subjects reversed the regimen, with those consuming a high-antioxidant diet consuming the supplement and vice versa. The postprandial sampling time of $1 \mathrm{~h}$ was chosen because several other studies (for examples, see Maxwell et al. 1994; Day \& Stansbie, 1995; Cao et al. 1998; Benzie et al. 1999) detected increased plasma antioxidant activity after similar periods. Compliance with the dietary regimen was assessed by interview with a dietitian, using a modified food intake questionnaire as a prompt at each visit. Consumption of the product was monitored by a brief questionnaire and only two of the subjects reported missing the supplement for more than $1 \mathrm{~d}$.

Informed consent was obtained from each volunteer, and the CSIRO Health Sciences and Nutrition Human Ethics Committee approved all procedures.

\section{Laboratory analyses}

Immediately after collection blood samples were placed on ice in the dark, and plasma was separated from the whole blood within $1 \mathrm{~h}$ and snap-frozen in liquid $\mathrm{N}_{2}$ before storage at $-80^{\circ} \mathrm{C}$. Antioxidant activity in plasma was determined by the method of Miller et al. (1993). Deproteinisation of the plasma was carried out by ultrafiltration (Centrisort 1, 20000d; Sartorius, Melbourne, Victoria, Australia). Metmyoglobin and 2,2'-azinobis(3ethylbenzothizoline-6-sulfonic acid (diammonium salt) and $\mathrm{H}_{2} \mathrm{O}_{2}$ were obtained from Sigma Chemicals (Sydney, NSW, Australia). Trolox was purchased from Aldrich Chemicals, Sydney, NSW, Australia. Ascorbic and uric acids were measured by HPLC following stabilisation of the plasma with metaphosphoric acid immediately after isolation (Nagy \& Degrell, 1989). Analysis of the plasma samples for antioxidants was carried out with slight modifications to the procedures described by Yang \& Lee (1987) and Khachik et al. (1992). A Shimadzu LC 10
HPLC (Shimadzu, Kyoto, Japan) fitted with a refrigerated autosampler and an SPD-M10Avp photodiode array detector with a class LC10 chromatography workstation was used for analysis of the prepared samples. Isocratic separations of the fat-soluble vitamins and carotenoids were carried out on a Rainin (Walnut CK, CA, USA) (4.6 mm i.d. $\times 250 \mathrm{~mm}$ length) $\mathrm{C}_{18}(5 \mu$ spherical particles) reverse-phase column. The mobile phase was a mixture of $(\%, \mathrm{v} / \mathrm{v})$ : acetonitrile 55, methanol 22, hexane 11.5 , dichloromethane 11.5 ; the flow rate was $1.0 \mathrm{ml} / \mathrm{min}$. Ammonium acetate $(0.01 \%, \mathrm{w} / \mathrm{v})$ was added to the mobile phase for stabilisation of the carotenoids. Trans- $\alpha-$ and $\beta$-carotene, lycopene, lutein, retinol, $\alpha$-tocopherol and $\alpha$-tocopheryl acetate were all obtained from Sigma Chemicals. Solvents, hexane, methanol, acetonitrile, ethanol and dichloromethane were all of analytical HPLC grade. A standard reference material (National Institute of Standards and Technology product 968b) was analysed with each batch of samples. The reference material with certified concentrations of retinol, $\alpha$-tocopherol, lutein and $\beta$-carotene was used to monitor the daily variation in analyses. The CV for the analyses ranged from $2.45 \%$ for retinol to $2.9 \%$ for $\alpha$-tocopherol. Statistics were evaluated by paired Student's $t$ test with $P=0.05$ as the criterion for significance.

\section{Results}

Concentrations of antioxidants present in the plasma are presented in Table 3. Ascorbic acid concentrations in the plasma samples taken after consumption of the antioxidantdepleted diet for 2 weeks were significantly lower $(P<$ $0.0001)$ than those of the other two dietary treatments. The concentrations of $\alpha$-carotene, $\beta$-carotene and lutein were all significantly increased $(P<0.005$ or less $)$ over the baseline concentrations following consumption of either the high-antioxidant diet or the supplement. Concentrations of $\alpha$-tocopherol, lycopene and retinol were largely unchanged during the course of the study, although there was a statistically, but probably non-physiologically, significant increase in fasting plasma retinol concentrations between the low-antioxidant dietary period and the highantioxidant dietary period $(P=0 \cdot 03)$. Plasma uric acid concentrations were unchanged during the study, but plasma albumin concentrations were significantly lower than those after either the high-antioxidant dietary period $(P<0.09)$ or consumption of the supplement $(P<0.004)$.

Plasma antioxidant activities from whole and deproteinised plasma samples collected after the overnight fast and $1 \mathrm{~h}$ after the breakfast are presented in Table 4 . There were no significant differences between any of the groups, either before or after the light breakfast or $15 \mathrm{~g}$ supplement. Removal of protein, which is a significant contributor to the antioxidant capacity of plasma, had no effect on antioxidant activity (Table 4) in the postprandial samples. Owing to insufficient material, deproteinised fasting plasma samples could not be analysed.

\section{Discussion}

As one of the major aims of the study was to monitor changes against a background diet low in antioxidants, 
Table 3. Antioxidants in plasma* from volunteers following 2-week period of consumption of low-antioxidant diets, diets high in antioxidants or the low-antioxidant diet together with $30 \mathrm{~g}$ dietary supplement $\dagger$

\begin{tabular}{lccccc}
\hline & $\begin{array}{c}\text { Low-antioxidant } \\
\text { diet }\end{array}$ & $\begin{array}{c}\text { High-antioxidant } \\
\text { diet }\end{array}$ & $\begin{array}{c}\text { Statistical significance } \\
\text { of difference: } P<\end{array}$ & $\begin{array}{c}\text { Statistical significance } \\
\text { of differenceł: } P<\end{array}$ \\
Pooled SE
\end{tabular}

* Fasting blood samples were collected after an overnight fast.

† For details of diets and supplement, see Tables 1 and 2 and p. 460, and for details of procedures, see p. 460.

‡ Relative to low-antioxidant diet.

baseline samples (i.e. samples collected whilst the subjects consumed their usual diets) were not collected. Following the adoption of a largely self-selected antioxidant-poor diet for 2 weeks, transition to a diet rich in fruits and vegetables resulted in significant increases in plasma concentrations of vitamin $\mathrm{C}$ and some, but not all carotenoids. Samman et al. (1997) showed significant reductions in plasma vitamin C concentrations after 2 weeks on a low-vitamin C dietary regimen. The observation that plasma albumin concentrations were significantly lower after the depletion period than after either supplementation period is difficult to interpret.

Rock et al. (1992) monitored depletion of carotenoids in plasma of subjects consuming diets low in carotenoids for up to 2 months. In their study plasma carotenoids, in particular lycopene, declined most rapidly over the first 2 weeks of the depletion period, followed by a slower reduction over the remaining period. From their results, they suggested that carotenoids exist in two pools in the body; one with rapid turnover, and another (possibly in tissue stores) with greater resistance to depletion. In the

Table 4. Antioxidant (AO) activities ( $\mu \mathrm{mol} 6$-hydroxy-2,5,7,8-tetramethylchroman-2-carboxylic acid equivalents/l) of whole and deproteinised plasma samples of volunteers following 2 weeks of consumption of low-AO diets, diets high in antioxidants or the low$\mathrm{AO}$ diet together with $30 \mathrm{~g}$ of the dietary supplement. ${ }^{*} \dagger$

(Mean values with their standard errors for twenty-five volunteers per treatment group)

\begin{tabular}{llllll}
\hline & \multicolumn{2}{c}{ Fasting } & & \multicolumn{2}{c}{ Postprandial } \\
\cline { 2 - 3 } \cline { 5 - 6 } & Mean & SE & & Mean & SE \\
\hline Whole plasma & & & & \\
$\quad$ Low-AO diet & 1.54 & 0.05 & & 1.52 & 0.05 \\
High-AO diet & 1.51 & 0.05 & & 1.50 & 0.06 \\
$\quad$ Supplement & 1.51 & 0.05 & & 1.51 & 0.06 \\
Deproteinised plasma & & & & 0.56 & 0.02 \\
$\quad$ Low-AO diet & ND & & & 0.56 & 0.03 \\
$\quad$ High-AO diet & ND & & & 0.56 & 0.03 \\
$\quad$ Supplement & ND & & & 0.56 & 0.0 \\
\hline
\end{tabular}

$\mathrm{ND}$, not determined.

* Fasting blood samples were collected after an overnight fast and the postprandial samples $1 \mathrm{~h}$ after consumption of toast and water (low- and high-AO diets) or $15 \mathrm{~g}$ of the supplement.

† For details of diets and supplement, see Tables 1 and 2 and p. 460, and for details of procedures, see p. 460. present study the dietary supplement contained little lycopene, so it is surprising that subjects consuming the supplement directly after the low-antioxidant period showed no change in their plasma lycopene concentrations. Indeed, plasma lycopene concentrations remained constant throughout the study, even when the participants were consuming a self-selected high-antioxidant diet. In addition, ascorbic acid concentrations were not different when the subjects consumed their chosen high-antioxidant diet or the supplement, despite the fact that the ascorbate content of the supplement was minimal. It is possible that inclusion of extra antioxidants in the diet via the supplement allowed ascorbate to be spared. Other studies have reported increased plasma antioxidant activity in periods ranging from less than $1 \mathrm{~h}$ up to $4 \mathrm{~h}$ following consumption of tea or tea polyphenols (Serafini et al. 1996; Benzie et al. 1999; Nakagawa et al. 1999), red wine or grape extracts (Maxwell et al. 1994; Day \& Stansbie, 1995; Whitehead et al. 1995; Cao et al. 1998; Durak et al. 1999). Cao et al. (1998) also reported increased plasma antioxidant activity following consumption of strawberries, spinach and vitamin C. The extract used in the present study contained antioxidants equivalent to approximately five to seven servings of fruit and vegetables per $d$. On the last day of the intervention with the supplement, antioxidant activities of plasma taken $1 \mathrm{~h}$ after consumption of the equivalent of 2.5-3.5 servings of fruit and vegetables was unchanged from the sample obtained after the overnight fast. Despite the measurable lower concentrations of antioxidants in the plasma after the depletion period, the antioxidant activity of the plasma samples did not increase after 2 weeks of dietary change or consumption of the extract. After removal of plasma albumin, which represents a major contributor to the total antioxidant capacity of plasma (Cao $\&$ Prior, 1998), the antioxidant capacity due to the lowermolecular-weight components did not change. Of the limited number of similar studies involving dietary manipulation over significant periods of time, Young et al. (1999) failed to find any increase in the antioxidant capacity of plasma measured either as the $\mathrm{Fe}^{3+}$-reducing ability of plasma (Benzie \& Strain, 1996) or as Troloxequivalent antioxidant capacity (Miller et al. 1993). Castenmiller et al. (1999) also failed to find any changes in antioxidant capacity in subjects supplemented with either 
$\beta$-carotene or spinach-based products, even though significant elevations in plasma carotenoid concentrations were detected. Cao et al. (1998) have compared the Troloxequivalent antioxidant capacity assay as described by Miller et al. (1993) and commercialised by Randox with two other methods, the $\mathrm{Fe}^{3+}$-reducing ability of plasma assay (described by Benzie \& Strain, 1996) and the oxygen radical absorbance assay developed by Cao et al. (1995). In their hands the Trolox-equivalent antioxidant capacity assay was less sensitive than either of the other assays to changes in plasma antioxidant activity in the $4 \mathrm{~h}$ following consumption of antioxidant foods. Differences between the methodologies of the assays might account for the lack of effect seen in the present study, although it is possible that the antioxidant capacity of plasma is under tight homeostatic control and can be overridden only with acute consumption of large amounts of antioxidant-rich foods or beverages.

\section{Conclusions}

Consumption of a dietary supplement representing antioxidants from five to seven servings of fruit and vegetables has been shown to increase the major antioxidant nutrients in human plasma to concentrations similar to those obtained through inclusion of fruits and vegetables in the diet. Lycopene and ascorbic acid concentrations were maintained or increased, even though the amounts supplied by the supplement were minimal. Despite the significant changes in plasma antioxidant components, there were no detectable changes in plasma antioxidant activity detectable by the Trolox-equivalent antioxidant capacity assay.

\section{Acknowledgements}

The authors wish to thank Dr Bob Steele and Mr Jim Culhane of Food Science Australia for their efforts in spray drying the vegetables. We also wish to thank Ms Manny Noakes for dietary advice and Sr Rosemary McArthur and the staff of the CSIRO Nutrition Clinic for their efforts, the volunteers for their enthusiasm and Dr Alex Jozsa from Sigma Pharmaceuticals. This study was supported in part by Sigma Pharmaceuticals (Melbourne, Victoria, Australia).

\section{References}

Ames BN (1998) Micronutrients prevent cancer and delay aging. Toxicology Letters 102-103, 5-18.

Ascherio A, Rimm EB, Giovanucci EL, Colditz GA, Rosner B, Willett WC, Sacks F \& Stampfer MJ (1992) A prospective study of nutritional factors and hypertension among US men. Circulation 86, 1475-1484.

Baghurst KI, Hertzler AA, Record SJ \& Spurr C (1992) The development of a simple dietary assessment and educational tool for use by individuals and nutrition educators. Journal of Nutrition Education 24, 165-172.

Benzie IFF \& Strain JJ (1996) The ferric reducing ability of plasma (FRAP) as a measure of 'antioxidant power': the FRAP assay. Analytical Biochemistry 239, 70-76.

Benzie IFF, Szeto YT, Strain JJ \& Tomlinson B (1999) Consumption of green tea causes rapid increase in plasma antioxidant power in humans. Nutrition and Cancer 34, 83-87.
Cao G \& Prior RL (1998) Comparison of different analytical methods for assessing total antioxidant capacity of human serum. Clinical Chemistry 44, 1309-1315.

Cao G, Russell RM, Lischner N \& Prior RL (1998) Serum antioxidant capacity is increased by consumption of strawberries, spinach, red wine or vitamin C in elderly women. Journal of Nutrition 128, 2383-2390.

Cao G, Verdon CP, Wu AHB, Wang H \& Prior RL (1995) Automated oxygen radical absorbance capacity assay using the COBAS FARA II. Clinical Chemistry 41, 1738-1744.

Castenmiller JJM, Lauridsen ST, Dragsted LO, van het Hof KH, Linssen JPH \& West CE (1999) $\beta$-Carotene does not change markers of enzymatic and nonenzymatic antioxidant activity in human blood. Journal of Nutrition 129, 2162-2169.

Day A \& Stansbie D (1995) Cardioprotective effect of red wine may be moderated by urate. Clinical Chemistry 41, 1319-1320.

Durak I, Köseoglu MH, Kaçmaz M, Büyükkoçak S, Çimen MYB \& Öztürk HS (1999) Black grape enhances plasma antioxidant potential. Nutrition Research 19, 973-977.

Gillman MW, Cupples LA, Gagnon D, Posner BM, Ellison RC, Castelli WP \& Wolf PA (1995) Protective effects of fruits and vegetables on stroke in men. Journal of the American Medical Association 273, 1113-1117.

Holland B, Welch AA, Unwin ID, Buss DH, Paul AA \& Southgate DA (1995) McCance and Widdowson's The Composition of Foods, 5th ed. p. 11. London: Royal Society of Chemistry and Ministry of Agriculture, Fisheries and Food.

Khachik F, Beecher GR \& Goli MB (1992) Separation and identification of carotenoids and their oxidation products in the extracts of human plasma. Analytical Chemistry 64, 21112122.

Lampe JW (1999) Health effects of vegetables and fruit: assessing mechanisms of action in human experimental studies. American Journal of Clinical Nutrition 70, 475S-490S.

Leske MC, Chylack LT, He Q, Wu SY, Schoenfeld E, Friend J \& Wolfe J (1998) Antioxidant vitamins and nuclear opacities: the longitudinal study of cataract. Ophthalmology 105, 831-836.

Maxwell S, Cruickshank A \& Thorpe G (1994) Red wine and antioxidant activity in serum. Lancet 344, 193-194.

Miller NJ, Rice-Evans CA, Davies MJ, Gopinathan V \& Milner A (1993) A novel method for measuring antioxidant capacity and its application to monitoring the antioxidant status in premature neonates. Clinical Science 84, 407-412.

Nagy E \& Degrell I (1989) Determination of ascorbic acid and dehydroascorbic acid in plasma and cerebrospinal fluid by liquid chromatography with electrochemical detection. Journal of Chromatography 497, 276-281.

Nakagawa K, Ninomiya M, Okubo T, Aoi N, Juneja LR, Kim M, Yamanaka K \& Miyazawa T (1999) Tea catechin supplementation increases antioxidant capacity and prevents phospholipid hydroperoxidation in plasma of humans. Journal of Agricultural and Food Chemistry 47, 3967-3973.

National Health and Medical Research Council (1991) Recommended Dietary Intakes for Use in Australia. Canberra: Australian Government Publishing Service.

Record IR, McInerney JK \& Record SJ (1998) Antioxidants and polyphenols in Australian foods. Proceedings of the Nutrition Society of Australia 22, 282.

Rimm EB, Ascherio A, Giovanucci E, Spiegelman D, Stampfer MJ \& Stalenhoef AH (1996) Vegetable, fruit and cereal fibre intake and risk of coronary heart disease among men. Journal of the American Medical Association 275, 447-451.

Rock CL, Swendseid ME, Jacob RA \& McKee RW (1992) Plasma carotenoid levels in human subjects fed a low carotenoid diet. Journal of Nutrition 122, 96-100.

Samman S, Brown AJ, Beltran C \& Singh S (1997) The effect of 
ascorbic acid on plasma lipids and oxidisability of LDL in male smokers. European Journal of Clinical Nutrition 51, 472-477.

Seddon JM, Ajani UA, Sperduto RD, Hiller R, Blair N, Burton TC, Farber MD, Gragoudas ES, Haller J, Miller DT, Yannuzzi LA \& Willett W (1994) Dietary carotenoids, vitamins A, C, and E, and advanced age-related macular degeneration. Eye Disease Case-Control Study Group. Journal of the American Medical Association 272, 1413-1420.

Serafini M, Ghiselli A \& Ferro-Luzzi A (1996) In vivo antioxidant effect of green and black tea in man. European Journal of Clinical Nutrition 50, 28-32.Steinmetz KA \& Potter JD (1996) Vegetables, fruit, and cancer prevention: a review. Journal of the American Dietetic Association 96, 1027-1039.
Whitehead TP, Robinson D, Allaway S, Syms J \& Hale A (1995) Effect of red wine ingestion on the antioxidant capacity of serum. Clinical Chemistry 41, 32-35.

Yang CS \& Lee M-J (1987) Methodology of plasma retinol, tocopherol, and carotenoid assays in cancer prevention studies. Journal of Nutrition, Growth and Cancer 4, 19-27.

Young JF, Nielsen SE, Haraldsdóttir J, Daneshvar B, Lauridsen ST, Knuthsen P, Crozier A, Sandström B \& Dragsted LO (1999) Effect of fruit juice intake on urinary quercetin excretion and biomarkers of antioxidative status. American Journal of Clinical Nutrition 69, 87-94. 